\title{
Is a Lag-Storage Curve an Early Sign of Diabetes?
}

\author{
Early Insulin Responses to i.v. Glucose in Normal Subjects, Mild Maturity-Onset Diabetics \\ and Patients with Lag-Storage Curves*
}

\author{
E. de Nobel, A. van 't Laar and Th. J. Benraad \\ Department of Internal Medicine, Radboud Ziekenhuis, University of Nijmegen, Nijmegen, The Netherlands
}

Summary. Early insulin responses were measured after a high dose $\left(50 \mathrm{~g} / 1.73 \mathrm{~m}^{2}\right)$ of rapidly injected glucose in 31 subjects who had repeatedly shown "lagstorage" curves in the OGTT, in 24 controls and in 19 mild maturity-onset diabetics. Division between controls and diabetics was virtually complete, when the insulin responses were expressed as "insulinogenic index" $\left(\frac{\Delta \text { insulin }}{\Delta \text { glucose }}\right)$. Twenty out of 31 patients with lag curves showed normal early insulin responses and 11 patients showed diabetic responses. In patients with lag curves, presence of obesity, and absence of family history of diabetes were associated with normal insulin responses. It is concluded that the finding of a lag curve is of little consequence in obese persons but, when in conjunction with a genetic background of diabetes, is suggestive of diabetes.

Key words: Initial glucose-induced insulin secretion, oral glucose tolerance test, intravenous glucose tolerance test, borderline glucose tolerance, maturity-onset diabetes, insulinogenic index, oxyhyperglycaemic curve, lag-storage curve.

The oral glucose tolerance test in patients with hyperthyroidism or partial gastrectomy frequently shows a pattern in which the blood glucose concentration rapidly rises to an abnormally high peak but falls to about the fasting level within two hours. This so-called "lag-storage" curve or "oxyhyperglycaemia" also occurs in healthy people. The prevalence in the normal

* Part of this material was presented at the 8th meeting of the EASD in Madrid, 1972 (24) population is estimated at approximately $9 \%$ [3]. The significance of this "idiopathic" lag-storage curve (or lag curve) remains obscure.

Some investigators $[22,23]$ regard the lag curve as a trivial abnormality; others $[1,9,16,20]$ however, have observed frequent progression to asymptomatic or symptomatic diabetes in patients exhibiting such curves and therefore regard the lag curve as an early sign of diabetes. Insulin release in patients showing lag curves is claimed to be delayed during oral glucose tolerance tests, when the release is compared to that observed in normals $[11,15]$ or in subjects with partial gastrectomy [19]. The insulinogenic index during the initial stage of the oral test in these patients is low as compared to normals [30]. Patients with lag curves have this phenomenon in common with mild diabetics. Furthermore a minority of them also have an intravenous glucose tolerance in the diabetic range [16, 19]. So there are several arguments indicating a lag curve as a diabetic feature. Definite evidence supporting such a view is still lacking, however.

In normal subjects intravenous administration of glucose is followed by a biphasic secretion of insulin $[5,26]$. The initial insulin response to intravenous glucose is blunted or absent in patients with mild maturity-onset diabetes $[10,27]$ and also in groups of subjects with prediabetes $[4,5,28]$. Diminished initial insulin secretion is therefore regarded as the earliest detectable sign of diabetes, or even as its "genetic marker" [6].

To examine the question whether patients with lag curves must be considered diabetics, early insulin responses to intravenous glucose were studied in patients with lag curves. For comparison results of similar investigations in a group of normal controls and a group of mild maturity-onset diabetics are also presented. 
Table 1. Subjects

\begin{tabular}{lcll}
\hline Group & $n$ & $\begin{array}{l}\text { Age } \\
\text { mean } \\
\text { (range) }\end{array}$ & $\begin{array}{l}\text { \% of ideal } \\
\text { bodyweight } \\
\text { (mean } \pm \text { SD) }\end{array}$ \\
\hline Controls & 24 & $\begin{array}{l}36 \\
(24-69)\end{array}$ & $109 \pm 11$ \\
$\begin{array}{l}\text { Patients with } \\
\text { lag curves }\end{array}$ & 31 & $\begin{array}{l}43 \\
(21-68)\end{array}$ & $116 \pm 17$ \\
Diabetics & 19 & $\begin{array}{l}48 \\
(18-63)\end{array}$ & $125 \pm 22$ \\
\hline
\end{tabular}

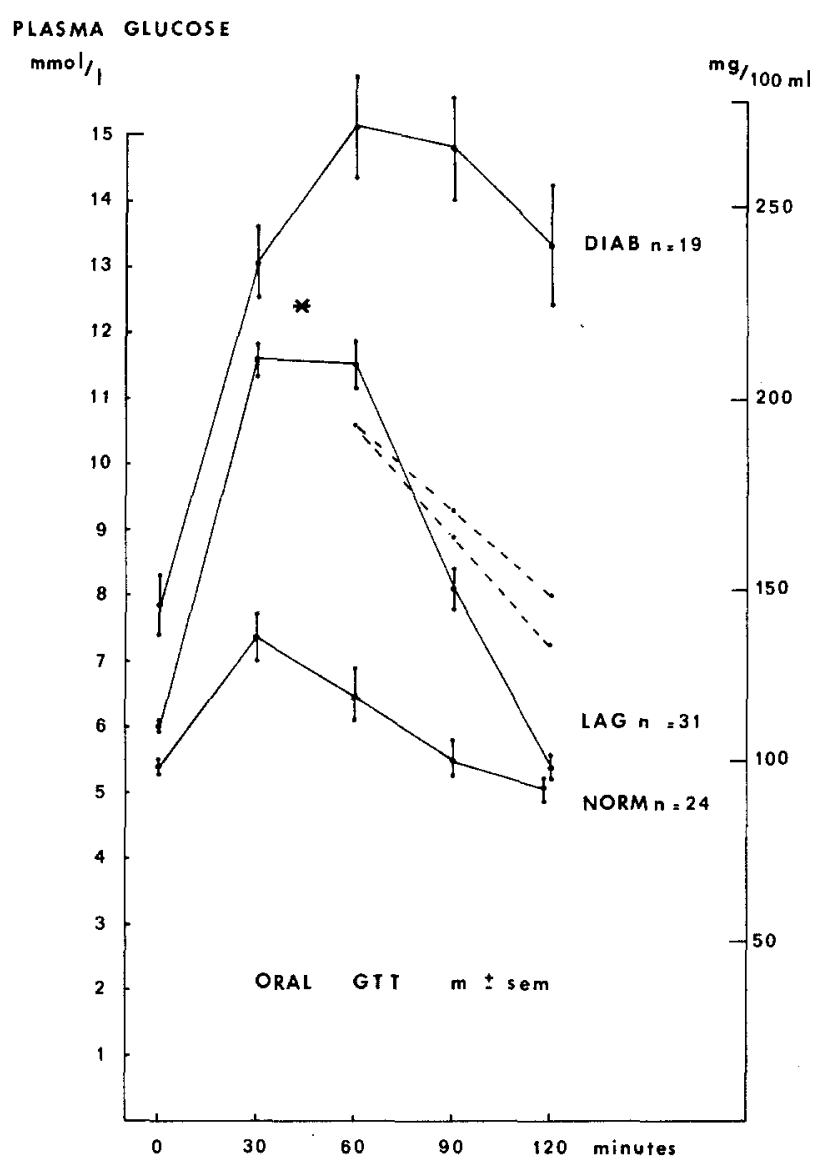

Fig. 1. Mean oral glucose tolerance curves of 19 mild maturity-onset diabetics, 31 patients with lag curves and 24 normal controls. Plasma glucose concentrations can be read in $\mathrm{mmol} / \mathrm{lor} \mathrm{mg} / 100 \mathrm{ml}$. The dashed lines represent the criteria of Fajans and Conn (14). The asterix denotes the mean peak value in the lag curve group, irrespective of time of occurrence

\section{Materials and Methods}

\section{Subjects}

The distribution of age and relative body weight in the three groups of subjects investigated is presented in Table 1. Selection of subjects was based on the re- sults in a standard $100 \mathrm{~g}$ oral glucose tolerance test (OGTT). The glucose curves obtained in the oral tests in the three groups are represented in Figure 1.

The 24 normal controls were selected because of their normal glucose tolerance as judged by the criteria of Fajans and Conn, adapted to the determination of glucose in plasma [14], and absence of family history of diabetes. Routine history and physical and biochemical examination revealed no abnormalities. Obesity was no reason for exclusion, but as a group the controls were not overweight. In seven subjects the body weight was more than $15 \%$ above the calculated ideal value [29].

An OGTT response was considered a lag curve when the plasma glucose peak at either 30 or $60 \mathrm{~min}$ after the $100 \mathrm{~g}$ load reached or was above $200 \mathrm{mg} / 100$ $\mathrm{ml}(11 \mathrm{mmol} / \mathrm{l})$ and the 2-hour-level was below 130 $\mathrm{mg} / 100 \mathrm{ml}(7.2 \mathrm{mmol} / \mathrm{l})$. Only those persons who had shown lag curves on at least two occasions within one month and had never shown other responses were selected. Patients with peptic ulcers, signs of hyperthyroidism or with partial gastrectomy were excluded. Screening of approximately 2100 OGTT's, performed in out-patients, resulted in the selection of 31 patients fulfilling these criteria and able and willing to undergo an IVGTT. Thirteen of these 31 subjects were obese, i.e. more than $115 \%$ of ideal weight [29]. First degree relatives with diabetes were known in 14 cases.

For the purpose of this study, diabetes was diagnosed if the $2 \mathrm{~h}$ glucose level in the OGTT was over $180 \mathrm{mg} / 100 \mathrm{ml}(10 \mathrm{mmol} / \mathrm{l})$ and the $\mathrm{K}$-value in a high dose intravenous glucose tolerance test [8] was below 1.3 , the critical value in this test being 1.5 . Thus cases of borderline glucose tolerance were excluded. Only untreated maturity-onset diabetics without a history of ketosis were included. None of them needed insulin later on. Of the 19 diabetic patients selected, 7 had overt disease as evidenced by fasting plasma glucose values of $8 \mathrm{mmol} / 1$ or more, the remaining 12 exhibiting merely "chemical" diabetes. Obesity as defined earlier was present in 10 individuals, 3 with overt and 8 with "chemical" diabetes. Differences in age between the three groups were not significant.

\section{Methods}

The standard OGTT was started at 8 a.m. after an overnight fast. When the patient was on a restricted diet, he was primed with a diet containing at least $200 \mathrm{~g}$ of carbohydrate during 3 preceding days. The $100 \mathrm{~g}$ glucose load was consumed as a $25 \%$ solution within $5 \mathrm{~min}$. Venous blood samples were taken before and every $30 \mathrm{~min}$ up to two hours after the load. The subject was seated all the time. Samples were centrifuged immediately. Determinations of glucose 
in plasma were performed with the auto-analyser (Hoffman's ferricyanide method, 12).

The intravenous challenge was given under the same conditions (except for a supine position of the patient) and consisted of $50 \mathrm{~g}$ of glucose per $1.73 \mathrm{~m}^{2}$ body surface. This high dose [8] was injected as a $50 \%$ solution in exactly $3 \mathrm{~min}$. The start of the injection was called zero time. Blood samples were taken from an indwelling catheter in the opposite arm, provided with a heparin lock. Three samples were taken prior to the glucose injection and subsequently $1,2,3,4,6,8,10$, $12,15,20,30,40,50,60,90$ and 120 min after the start of the injection. Centrifuged aliquots of plasma for determination of insulin were stored at $-20^{\circ} \mathrm{C}$ until radioimmunoassay. Separation of the "free" and "bound" fractions was performed with a "double antibody solid phase" technique [13]. Glucose disappearance rates (K-values) were calculated from semilogarithmic plots of absolute plasma glucose values, according to the formula of Lundbaek [16]: $\mathrm{K}=$ $\frac{0.693}{t^{1 / 2}} \times 100$. For each experiment, an insulinogenic index [27] was calculated by dividing the fasting-topeak insulin rise $(\mu \mathrm{U} / \mathrm{ml})$ by the concomitant change in plasma glucose $(\mathrm{mmol} / \mathrm{l})$ :

$\Delta$ insulin. The insulin peak was invariably reached at $\Delta$ glucose

three or four minutes after the start of the injection.

For statistical analysis Wilcoxon's test (for unpaired and for paired observations) was used, and Spearman's correlation coefficient, as results did not represent normal distributions.

\section{Results}

\section{Plasma Glucose}

The mean plasma glucose concentration in the fasting state was slightly but significantly higher in patients with lag curves than in controls $(\mathrm{p}<0.02)$. This was not due to the presence within this group of subjects with significant hyperglycaemia: the highest fasting value encountered (in one extremely obese patient) was $7.05 \mathrm{mmol} / \mathrm{l}$. Fasting glucose levels were substantially higher in the diabetic group. The glucose peaks reached in the intravenous tests were not significantly different in the three groups: $30.2 \pm 4.3 \mathrm{mmol} / 1(\mathrm{M} \pm$ S.D.) in normals, $31.8 \pm 4.1$ in patients with lag curves and $32.4 \pm 4.1$ in diabetics.

The mean fasting-to-peak change of the plasma glucose in the i.v. test ( $\Delta$ glucose $)$ was $25.0 \mathrm{mmol} / 1$ for controls, $26.2 \mathrm{mmol} / 1$ for patients with lag curves and $25.2 \mathrm{mmol} / \mathrm{l}$ for diabetics. This insulinogenic stimulus was not statistically different in the three groups.

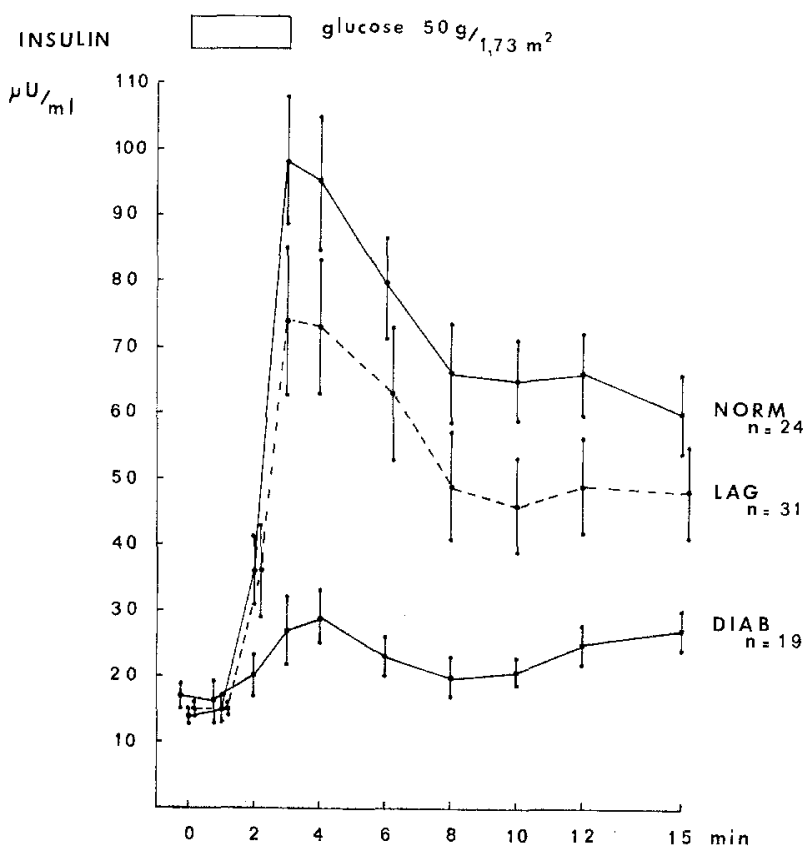

Fig. 2. Early insulin responses (mean \pm SEM) following a large intravenous glucose load rapidly injected in normal controls (NORM), patients with lag curves (LAG) and mild maturity-onset diabetics (DIAB). Differences are significant with $\mathrm{p}<0.05$ (NORM : LAG) and $p<0.005$ (LAG: DIAB)

\section{$K$-Values}

The glucose disappearance rates (K-values) in the normal controls all were greater than 1.5 , with a mean of $2.45 \pm 0.96$ (S.D.). These values were unduly influenced by a few individuals with very high $\mathrm{K}$ values, causing skewness of the distribution to the right. By definition, $\mathrm{K}$-values in diabetics were less than 1.3. In the lag-storage group, $\mathrm{K}$-values were normal i. e. greater than 1.5, in 16 cases, diabetic (less than 1.3) in 9 cases and equivocal (between 1.3 and 1.5 ) in the remaining 6 . The mean $\pm \mathrm{SD}$ for all lag curve patients was $1.58 \pm 0.42$, the difference from the control group being highly significant $(\mathrm{p}<0.005)$.

\section{Insulin Responses}

Mean insulin levels in the fasting state were not significantly different in the three groups: $14 \pm 4 \mu \mathrm{U} / \mathrm{ml}$ (S.D.) in the normals, $16 \pm 6$ in the patients with lag curves and $17 \pm 8$ in the diabetics. Early insulin responses (mean \pm SEM) to the intravenous glucose challenge are shown in Figure 2.

During the $15 \mathrm{~min}$ of the test depicted here, the mean response of the lag-storage group is less than that of the control group. As such, this is a significant phenomenon $(p<0.05$ in the sign test for 7 differences). When each time-point is considered separate- 


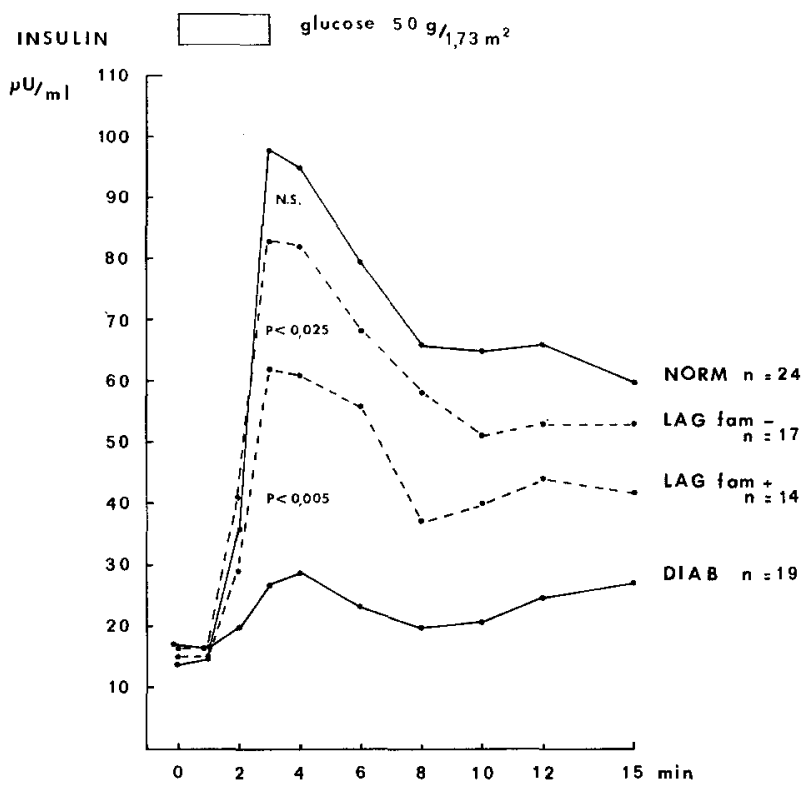

Fig. 3. Early insulin responses in the same subjects as represented in Fig. 2. However, the lag curve group has been divided into two subgroups: one with positive family history of diabetes (LAG fam + ), and a group without such family history (LAG fam -). P-values denote significance of differences between the peaks of the adjacent curves

Table 2. Insulin release, expressed as mean insulinogenic index, in the normal and the lag-storage groups, each divided according to obesity. The difference between the groups is confined to the nonobese categories

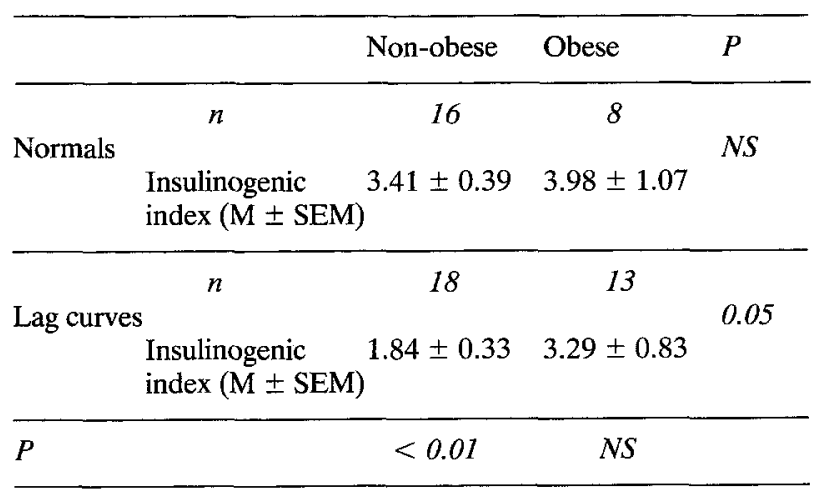

ly, differences between these two groups do not reach statistical significance, but the difference between the means of the peaks (irrespective of their occurrence at 3 or $4 \mathrm{~min}$ ) is significant at the $5 \%$ level. The mean insulin curve of all patients with lag curves resembles that of the normal group in exhibiting a steep early rise and is at all points significantly separated from the curve of the diabetics.

\section{Influence of Family History}

Figure 3 gives early insulin responses divided on the basis of negative or positive family history for dia-

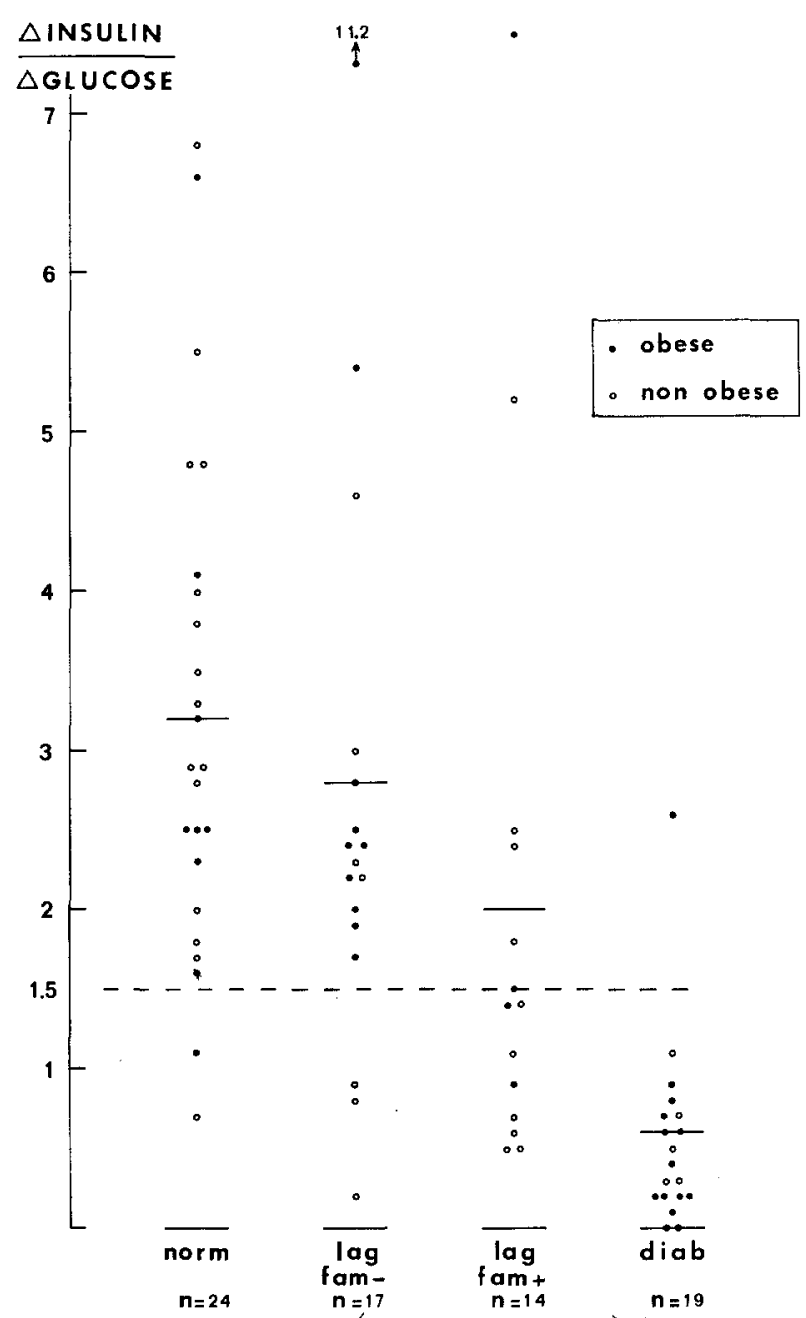

Fig. 4. Insulinogenic indices $\left(\frac{\Delta \text { insulin in } \mu \mathrm{U} / \mathrm{mI}}{\Delta \text { glucose in } \mathrm{mmol} / \mathrm{l}}\right)$ for early peaks after intravenous glucose, in normal controls (NORM), mild maturity-onset diabetics (DIAB) and patients with lag curves (LAG), with and without a family history of diabetes. Each point represents the index for one subject. Open circles stand for lean patients, dots for obese patients. The dashed line gives the best separation between normals and diabetics

betes. The mean insulin curve of the 17 patients with lag curves and no diabetes in their families is at all points lower than that of the control group, but the mean initial peaks do not differ significantly. However, the difference between the responses of the subgroups with and without a family history for diabetes is significant at 3, 4, 6 and $8 \mathrm{~min}$. The group with family history released significantly more insulin than did the diabetic group ( $\mathrm{p}<0.005$ for peaks).

\section{Insulin Responses of Individuals}

How individuals responded can be derived from Figure 4 , where the insulinogenic indices are shown for all 
subjects tested. There is nearly complete division between normals and mild maturity-onset diabetics at the value of 1.5 . The mean values ( \pm SEM) of the indices for the groups were $3.23 \pm 0.33$ for controls, $2.45 \pm 0.41$ for all patients with lag curves and $0.55 \pm$ 0.13 for diabetics. Statistically the differences between the means of the three groups were clearly significant $(\mathrm{p}<0.01)$.

Of the 31 patients with lag curves, 20 showed normal responses whereas 11 had blunted, diabeticlike, early insulin secretion (insulinogenic index < 1.5). Out of 17 patients showing lag curves without diabetes among their first degree relatives, only 3 exhibited low insulin responses (index $<1.5$ ).

\section{Relation of Insulin Response to K-Value}

Within the lag-storage group, glucose disappearance rates were not significantly correlated with the height of the early insulin peaks $(r=0.15)$. Insulinogenic indices were normal in 10 out of 16 patients with normal K-values, in 7 out of 9 with low and in 3 out of 6 with borderline $\mathrm{K}$-values.

When the 9 subjects with lag curves and subnormal, diabetic $\mathrm{K}$-values are discarded, the mean insulin peak of the remaining lag-patients is still lower than that of the controls, but with marginal significance $(0.05<\mathrm{p}<0.10)$. The mean insulinogenic index, however, remains definitely diminished in this group $(\mathrm{p}<0.02)$. There was no difference in relative bodyweight between both subgroups of patients with lag curves: $(118 \% \pm 18$ S.D. for the low-K-group and $115 \pm 17$ for the group of patients with normal K-value).

\section{Relation of Insulin Response to Obesity}

Within the lag-storage group, but not within the other groups, there was a significant correlation between insulin response and degree of obesity $(r=0.38 ; p<$ $0.05)$. When all cases with obesity $(115 \%$ or more of ideal bodyweight) are rejected, 18 patients with lag curves $(105 \% \pm 7$ S.D. $), 16$ controls $(102 \pm 5)$ and 9 diabetics $(109 \pm 4)$ remain to be considered. The relationships between these three groups are exactly the same as have been described for the whole groups, as far as mean insulin curves, mean insulin peaks and mean insulinogenic indices are concerned. Significance of the differences regarding the subgroups with normal and subnormal $\mathrm{K}$-values and with different family histories is lost however. In Table 2 mean insulinogenic indices are given for obese and non-obese subjects separately. It is obvious from this table that the difference in insulin release between the normal and the lag-storage group is confined to the non-obese category.
Twenty patients with lag curves could be matched, with regard to degree of obesity, with 20 of the controls. The matching procedure was performed without knowledge of $\mathrm{K}$-values or insulin secretion patterns, but age was known and taken into account whenever more than one possibility for matching occurred. Mean relative weights were identical in the two groups so formed: $110 \%$ of ideal \pm 14 S.D.; 7 pairs were obese. The difference, within pairs, of relative weight never exceeded $3 \%$ and was zero in 10 of them; the calculated duplicate variation was $1 \%$.

K-values were definitely subnormal in 7 out of 20 patients and marginal in 2 . The mean $\mathrm{K}$ for controls was $2.45 \pm 0.95 \mathrm{~S}$. D., for patients $1.54 \pm 0.42(\mathrm{p}<$ $0.01)$.

The mean insulin curve of the patients was at all timepoints situated below that of the controls. The mean peaks $( \pm$ S.D.) were $99 \mu \mathrm{U} / \mathrm{ml}( \pm 50)$ and 75 $( \pm 50)$ respectively $(0.05<\mathrm{p}<0.10)$. The means of the insulinogenic indices were: $3.37 \pm 1.68$ S.D. and $2.30 \pm 1.75$. The groups differed significantly in this respect $(\mathrm{p}<0.02)$. When pairs with a subnormal $\mathrm{K}$-value in the patient-partner are discarded, the significance of the difference between the indices of the groups becomes marginal $(0.05<p<0.10)$. When the 20 pairs are divided in two subgroups as to relative bodyweight over or below $115 \%$, it appears that only in non-obese pairs is there a significant difference in insulinogenic index between normals and patients with lag curves $(p<0.05)$. This difference is insignificantly small in obese pairs (mean difference $0.33 \pm$ 0.72 ).

\section{Discussion}

The present method of measuring early insulin responses to i.v. glucose loading is in some respects different from those used by most other investigators. Rapid injection of a high dose of glucose was used, as described by Dyck and Moorhouse [8]. These investigators have clearly shown [21] that high doses permit better distinction between glucose disappearance rates (K-values) of normals and diabetics. Rapid glucose injection yields higher insulin responses; even small differences in injection time cause striking differences in insulin response [10]. Phlebitis was seen only in three cases out of the nearly 150 tests performed so far. The necessity of early sampling is well established, both for i.v. tolbutamide [17] and i.v. glucose $[4,25]$ loading tests.

From Figure 4 it is obvious that this method not only permits good distinction between normal and diabetic groups, but also allows classification, with minimal overlap, of individuals as normal or diabetictype insulin secretors. 
Cerasi and coworkers [7] have demonstrated a dose-related insulin response to i.v. glucose: their "low-responders" approximated normal insulin secretion when the glucose challenge was very high. It could therefore be argued that the high dose we used lessened the discriminative power of our test. However, Cerasi and coworkers take the first blood sample 10 min after the start of the injection, whereas, as is again demonstrated in this study, insulin peaks occur earlier. Pfeiffer [25], using early sampling, did not find a dose-response relationship within the range described by Cerasi. Moreover, even in the highest blood-glucose range achieved by Cerasi, distinction between his low- and high-responders remains fairly good and the range obtained in the present investigation was substantially lower. And, finally, individuals with normal glucose tolerance and decreased early insulin secretion, deemed prediabetics by Luft and Cerasi, also appear to be detectable with the present method.

The finding of glucose disappearance rates (Kvalues) in the definitely diabetic range in 9 out of 31 patients with lag curves is in accordance with the 3 out of 11 reported by Lundbaek [16] and the 2 out of 6 found by McKiddie et al. [19]. As the intravenous test generally is regarded as less sensitive than the oral G.T.T. [18], these results can be explained as evidence that a lag curve indicates decreased glucose tolerance.

Within the lag-storage group, no correlation could be found between oral glucose tolerance (the sum of the 5 values taken as a parameter) or intravenous glucose tolerance ( $\mathrm{K}$-value) and the insulinogenic index. This latter finding is at variance with the work of Porte and Pupo [26] with small doses. An observation similar to ours however, was made by Alford and coworkers [2] in a group of patients with mildly abnormal oral glucose tolerance, also challenged with a high intravenous glucose dose. Apparently, decreased early insulin secretion does not simply reflect the decrease in glucose tolerance. This underscores the hypothesis of Cerasi and Luft [6] on the specific meaning of blunted early insulin secretion as a sign of prediabetes.

A lower than normal mean insulin response to intravenous glucose in patients with lag curves has briefly been reported by McKiddie and coworkers [19]. The individual results of their 9 subjects are not given and the finding is not discussed. In the present study, the occurrence of a lag curve was, in about one third of cases, accompanied by sluggish early insulin secretion as a marker of impending or incipient diabetes. Even when we removed those individuals who appeared to be diabetic because of their subnormal $\mathrm{K}$-values, this procedure did not substantially alter the ratio ( 9 out of 22 ).
The results cannot be attributed to the differences between groups in degree of obesity. On the contrary, one would expect a possibly decreased insulin secretion in the group of patients with lag curves to be obscured rather than caused by their higher mean weight. Table 2 illustrates this point. Procedures meant to rule out the influence of obesity (i. e. discarding of all overweight persons, or matching for relative weight) do not change the results, but they reduce the number of subjects under investigation. With the wide variation of insulin responses, the decreased statistical significance obtained should be explained this way.

The insulin response separates patients with lag curves, but without first degree relatives with diabetes, more clearly from those with such family history, than from normal controls (Figs. 3 and 4). This could mean that a lag curve in conjunction with a positive family history is indicative of diabetes.

When not accompanied by a strong family history of diabetes, a lag curve is indicative of early diabetes only in a small minority of cases. Obesity seems even further to decrease a possible specific significance of the lag curve: e.g. 10 obese persons with lag curves had normal early insulin secretion, whereas the 3 obese "low-responders" all had positive family histories.

The results presented here suggest that a lagstorage curve has a significance comparable to that of borderline or slightly diminished glucose tolerance. As such, the finding of a lag curve is of little consequence in obese persons. However, when associated with a genetic background of diabetes either in lean or obese patients, a lag curve is suggestive of diabetes.

\section{References}

1. Ackerman, I.P., Fajans, S.S., Conn, J.W.: The development of diabetes mellitus in patients with nondiabetic glycosuria. Clin. Res. Proc. 6, (Abstr.) 251 (1958)

2. Alford, F.P., Martin, F.I.R., Pearson, M.J.: The significance and interpretation of mildly abnormal oral glucose tolerance. Diabetologia 7, 173-180 (1971)

3. Birmingham Survey Working Party: Glucose tolerance and glycosuria in the general population. Brit. med. J. 1963 III, 655-659

4. Boden, G., Soeldner, J.S., Gleason, R. E., Marble, A.: Elevated serum human growth hormone and decreased serum insulin in prediabetic males after intravenous tolbutamide and glucose. $\mathrm{J}$. clin. Invest. 47, 729-739 (1968)

5. Cerasi, E., Luft, R.: Plasma-insulin response to sustained hyperglycaemia induced by glucose infusion in human subjects. Lancet 1967 II, 1359-1361

6. Cerasi, E., Luft, R.: "What is inherited - what is added" hypothesis for the pathogenesis of diabetes mellitus. Diabetes 16, 615-627 (1967)

7. Cerasi, E., Luft, R., Efendic, S.: Decreased sensitivity of the pancreatic beta cells to glucose in prediabetic and diabetic subjects. A glucose dose-response study. Diabetes 21, 224-234 (1972) 
8. Dyck, D. R., Moorhouse, J.A.: A high-dose intravenous glucose tolerance test. J. clin. Endocr. 26, 1032-1038 (1966)

9. Fajans, S.S., Conn, J.W.: The early recognition of diabetes mellitus. Ann. N. Y. Acad. Sci. 82, 208-218 (1959)

10. Fujita, Y., Herron, A.L., Seltzer, H.S.: Confirmation of impaired early insulin response to glycemic stimulus in non-obese mild diabetics. Diabetes 24, 17-27 (1975)

11. Goto, Y., Sato, S.-I., Ohira, S., Maruhama, Y.: Plasma insulin and free fatty acids in young people with postprandial glycosuria: plasma insulin response in the cases with borderline glucose tolerance test. Diabetologia 9, 264-267 (1973)

12. Hoffman, W.S.: A Rapid photoelectric method for the determination of glucose in blood and urine. J. biol. Chem. 120, 51-55 (1937)

13. Hollander, F.C. den, Schuurs, A.H.W.M., Hell, H. van: Radioimmunoassays for human gonadotrophins and insulin employing a "double-antibody solid-phase" technique. J. Immunol. Methods 1, 247 (1972)

14. Klimt, C. R., Prout, T. E., Bradley, R. F., Dolger, H., Fisher, G., Gastineau, C.F., Marks, H., Meinert, C. L., Schumacher, O.P.: Standardization of the oral glucose tolerance test. Diabetes 18, 299-307 (1969)

15. Kryston, L.J., Shaw, R.A., Molines, M., Mills, L.C.: Atypical glucose and insulin responses in prediabetes. Diabetes 20 (Suppl. 1), (Abstr.) 364 (1971)

16. Lundbaek, K.: Intravenous glucose tolerance as a tool in definition and diagnosis of diabetes mellitus. Brit. med. J. 1962 II, $1507-1513$

17. Maingay, D., Touber, J.L., Schopman, W., Ruyter, H.A. de, Croughs, R.J.M., Lequin, R.M.: Rapid rise of insulin concentration in the plasma after intravenous administration of sodium tolbutamide. Lancet 1967 I, 361-362

18. Marble, A., White, P., Bradley, R. F., Krall, L.P.: Joslin's diabetes mellitus, 11th Ed., p. 204. Philadelphia: Lea and Febiger 1971

19. McKiddie, M.T., Scott, R.T.A., Buchanan, K.D.: Plasma insulin studies in patients with lag curves. Diabetologia 6 , 420-424 (1970)

20. McKiddie, M.T., Scott, R.T. A., Cole, R.: The insulin response to glucose in patients with non-diabetic glycosuria. Postgrad. med. J. 47, 605-610 (1971)
21. Moorhouse, J. A., Steinberg, J., Tessler, B. B.: Effect of glucose dose upon intravenous glucose tolerance in health and in diabetes. J. clin. Endocr. 23, 1074-1079 (1963)

22. Mosenthal, H.O., Barry, E.: Criteria for and interpretation of normal glucose tolerance tests. Ann. Intern. Med. 33, 1175-1194 (1950)

23. Moyer, J.H., Womack, C. R.: Glucose tolerance: I. A comparison of 4 types of diagnostic tests in 103 control subjects and 26 patients with diabetes. Amer. J. med. Sci. 219, 161-173 (1950)

24. Nobel, E. de, Laar, A. van 't, Koene, R.A.P., Benraad, T.J.: Insulin response to intravenous glucose administration in normal subjects, mild maturity-onset diabetics and subjects with a "Lag-storage" curve. Diabetologia 9, (Abstr.) 65 (1973)

25. Pfeiffer, E.F.: Dynamics of insulin secretion in normal, obese and diabetic subjects following beta-cell stimulation. In: Tolbutamide . . . after ten years. pp. 127-139. Amsterdam: Excerpta Medica Internat. Congr. Series Nr. 149, 1967

26. Porte, D., Pupo, A. A.: Insulin responses to glucose: evidence for a two pool system in man. J. clin. Invest. 48, 2309-2319 (1969)

27. Seltzer, H.S., Allen, E.W., Herron, A.L., Brennan, M. T.: Insulin secretion in response to glycemic stimulus: relation of delayed initial release to carbohydrate intolerance in mild diabetes mellitus. J. clin. Invest. 46, 323-335 (1967)

28. Simpson, R.G., Benedetti, A., Grodsky, G.M., Karam, J.H., Forsham, P.H.: Early phase of insulin release. Diabetes 17, 684-692 (1968)

29. Statist. Bull. Metrop. Life Insur. Co. 40, 1-4 (1959)

30. Stephan, T., Nolan, S., Khurana, R.C., Morgan, C. R., Wingert, J.P., Danowski, T.S.: Lag glucose tolerance curves. Amer. J. med. Sci. 264, 41-47 (1972)

Received: May 7, 1976, and in revised form: October 14, 1976

Dr. E. de Nobel

Dept. of Internal Medicine

Radboud Ziekenhuis

University of Nijmegen

Geert Groote plein Zuid 16

Nijmegen

The Netherlands 